\title{
Joint Space Reference Trajectory to Reduce the Energy Consumption of a Six-Legged Mobile Robot
}

DOI:

10.1109/IWED.2018.8321378

\section{Document Version}

Accepted author manuscript

Link to publication record in Manchester Research Explorer

\section{Citation for published version (APA):}

Bodrov, A., Cheah, W., Green, P., Watson, S., \& Apsley, J. (2018). Joint Space Reference Trajectory to Reduce the Energy Consumption of a Six-Legged Mobile Robot. In 2018 25th International Workshop on Electric Drives: https://doi.org/10.1109/IWED.2018.8321378

\section{Published in:}

2018 25th International Workshop on Electric Drives:

\section{Citing this paper}

Please note that where the full-text provided on Manchester Research Explorer is the Author Accepted Manuscript or Proof version this may differ from the final Published version. If citing, it is advised that you check and use the publisher's definitive version.

\section{General rights}

Copyright and moral rights for the publications made accessible in the Research Explorer are retained by the authors and/or other copyright owners and it is a condition of accessing publications that users recognise and abide by the legal requirements associated with these rights.

\section{Takedown policy}

If you believe that this document breaches copyright please refer to the University of Manchester's Takedown Procedures [http://man.ac.uk/04Y6Bo] or contact uml.scholarlycommunications@manchester.ac.uk providing relevant details, so we can investigate your claim.

\section{OPEN ACCESS}




\title{
Joint Space Reference Trajectory to Reduce the Energy Consumption of a Six-Legged Mobile Robot
}

\author{
Alexey Bodrov, Weichen Cheah, Peter N. Green, Simon Watson, Judith Apsley \\ School of Electrical and Electronic Engineering \\ The University of Manchester \\ Manchester, M13 9PL, United Kingdom
}

\begin{abstract}
Energy consumption minimization is an essential issue for the mobile robots directly affecting their autonomy. This is particularly important for legged robots, which are less efficient than wheeled robots, but are more suitable for locomotion across irregular and inclined terrain. In this paper a novel strategy to control the legged mobile robot with reduced energy consumption is presented. It is based on reference trajectory optimization for each joint/actuator. Experimental results show the $23.6 \%$ energy consumption reduction compared to the conventional control approach.
\end{abstract}

Keywords—legged mobile robots; energy consumption; DC actuators; joint torques

\section{INTRODUCTION}

Mobile robots find applications in different areas of modern industry. The CORIN mobile robot in Fig. 1 has been developed as a mobile platform for remote inspection in hazardous environment. The obvious drawback of mobile robots compared with fixed robots is the lack of a mainsconnected power supply, so energy consumption is a primary issue for these types of robots, affecting battery size and operational range. Wheeled robots are the main type of land mobile robots [1], with applications ranging from low power household mobile cleaners [2] to car-sized robots for space exploration [3]. Compared to the more complex legged robots, wheeled robots are characterized by high efficiency, light weight and high speed, however wheeled robots have a number of significant drawbacks such as the requirement for a continuous path to travel on, coupled relations between the body path and the wheel path (lower stability of the upper body compared to the legged robot) and higher sensitivity to ground disturbance [4], [5]

Due to specific characteristics such as inclined irregular terrain in the nuclear power plants [6], the legged-type robot was preferred for the inspection role over the wheeled-type robots during the development of a project. There are plenty of possible designs of a legged robot; according to classification based on the leg number it could be biped, quadruped, octopod and so on, but the hexapod configuration has been chosen because it has the minimum number of legs for statically stable movement [7] and redundant configuration features, compared to the other designs.

During the past decades a number of optimization techniques have been developed in order to reduce the hexapod

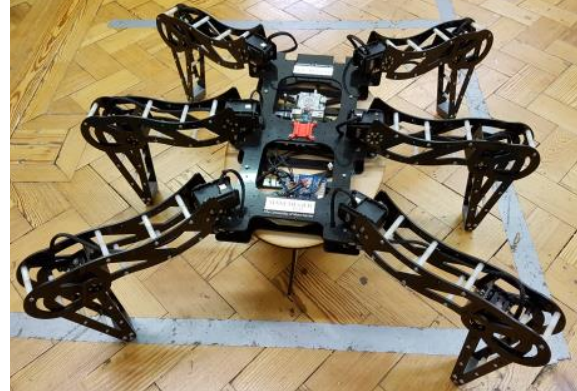

Fig. 1. CORIN mobile robot.

power consumption. Some authors focus on the efficient actuator design used in hexapod, where they try to increase the mechanical power output while keeping the size and weight as small as possible [8]. Others perform the optimization of a single leg structure or the way leg is connected to the hip (lateral connection versus vertical connection). In [9], the different leg structures presented in [10] are studied from an energy efficiency point of view. There is a big focus on robot energy efficiency optimization via the gait parameters. In [11] the rule for setting the velocity, footholds, step height, stroke and duty factor maximizing the energy efficiency is presented. [12], [13] show that a low duty factor is preferable for the wave gait from the energy-efficiency point of view for both straight and turning locomotion. In [14] switching between the different gait patterns according to the terrain is proposed. With preselected gaits [13] and [15] minimize the power consumption by optimising joint torques/feet forces distributions. It is shown that for the statically stable locomotion of the six-legged robot the distribution of feet forces or joint torques becomes indeterminate and could be a subject of optimization.

In this paper a different approach for the energy efficient control of the leg actuators is presented, which extends the loss analysis to the actuator motors. It is not refuting the aforementioned methods but provide an extension, where the consumed energy is minimized by modifying the actuator reference trajectories in the joint space.

The paper is structured as follows. First, the CORIN kinematics is described. Then the joint space trajectory modification is discussed in order to find one that minimizes the energy consumption. Further the experimental verification of the proposed method is shown and finally the discussion of the obtained results is presented. 

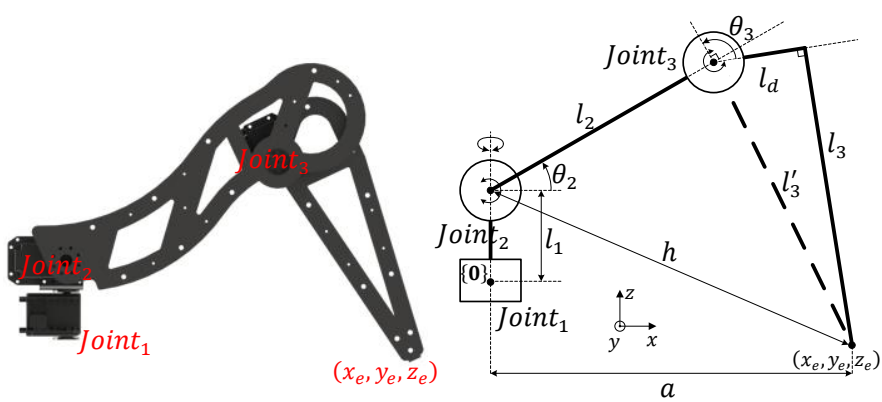

(a)

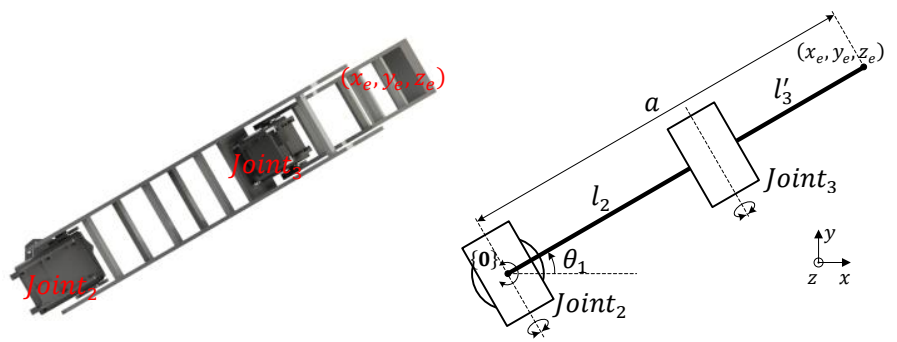

(b)

Fig. 2. CORIN leg geometry: a) Side view b) Top view.

\section{CORIN GEOMETRY}

This paper is focused on energy consumption minimization, where the leg actuators are controlled to follow the reference trajectory in the joint space (which will be discussed in further sections), but it is natural to specify the robot (each leg) position in the Cartesian space along the moving path. Therefore the transformation between the joint and Cartesian spaces is needed. In robotics applications, this is conventionally done through the forward and inverse kinematic transformations for joint to Cartesian and for Cartesian to joint spaces respectively. CORIN has a rectangular body shape, with each leg having three degrees of freedom (DOF) and each joint within a leg is powered by a geared DC motor. Each joint's shaft is equipped with an absolute optical encoder to measure the rotor speed and position. The side and top views of a single leg together with its schematic representation are shown on Fig. $2 \mathrm{a}$ and $2 \mathrm{~b}$ respectively and the robot parameters are summarized in Table I.

For a single CORIN leg by applying a similar procedure to [16], the forward kinematics using the Denavit-Hartenberg parameter for each axis $(x, y, z)$ could be derived as follows.

$$
\left[\begin{array}{l}
x_{e} \\
y_{e} \\
z_{e}
\end{array}\right]=\left[\begin{array}{c}
\cos \theta_{1}\left[l_{3} \cos \left(\theta_{2}+\theta_{3}\right)+l_{2} \cos \theta_{2}-l_{d} \sin \left(\theta_{2}+\theta_{3}\right)\right] \\
\sin \theta_{1}\left[l_{3} \cos \left(\theta_{2}+\theta_{3}\right)+l_{2} \cos \theta_{2}-l_{d} \sin \left(\theta_{2}+\theta_{3}\right)\right] \\
l_{1}+l_{3} \sin \left(\theta_{2}+\theta_{3}\right)+l_{2} \sin \theta_{2}+l_{d} \cos \left(\theta_{2}+\theta_{3}\right)
\end{array}\right]
$$

Using (1) the actual position of feet with respect to the body in the Cartesian space can be identified using the encoder readings from each joint.

The inverse kinematic equations showing the joint angles in relation with the Cartesian position can be obtained geometrically. For the given link sizes $\left(l_{i}\right)$ and other
TABLE I. CORIN DESIGN PARAMETERS

\begin{tabular}{|c|c|c|c|}
\hline \multicolumn{4}{|c|}{ Geometrical parameters } \\
\hline$l_{1},[\mathrm{~m}]$ & $l_{2},[\mathrm{~m}]$ & $l_{3},[\mathrm{~m}]$ & $l_{d},[\mathrm{~m}]$ \\
\hline 0.04475 & 0.192 & 0.192 & 0.028 \\
\hline \multicolumn{3}{|c|}{ Joint mass, [kg] } & Full body weight, [kg] \\
\hline$m_{1}$ & $m_{2}$ & $m_{3}$ & $M$ \\
\hline 0.072 & 0.126 & 0.072 & 4.7 \\
\hline
\end{tabular}

geometrical parameters given in Fig. 2, joint rotation angles $\theta_{i}$ are obtained by applying the following equalities.

$$
\begin{aligned}
& \theta_{1}=\tan ^{-1} \frac{y_{e}}{x_{e}} \\
& \omega=\tan ^{-1} \frac{l_{1}-z_{e}}{a} \\
& \left.\beta=\cos ^{-1}\left(\frac{l_{3}^{\prime 2}-l_{2}^{2}-h^{2}}{2 l_{2} h}\right)\right\} \quad \theta_{2}=\beta-\omega \\
& l_{3}^{\prime}=\sqrt{l_{3}^{2}+l_{d}^{2}} \quad c=\tan ^{-1} \frac{l_{3}}{l_{d}} \\
& \left.\begin{array}{c}
a=\sqrt{x_{e}^{2}+y_{e}^{2}} \\
h=\sqrt{a^{2}+\left(z_{e}+l_{1}\right)^{2}} \\
\alpha=\cos ^{-1}\left(\frac{h^{2}-l_{2}^{2}-l_{3}^{2}}{2 l_{2} l_{3}^{\prime}}\right)
\end{array}\right\} \theta_{3}=\frac{3 \pi}{2}-\alpha-c
\end{aligned}
$$

\section{OPTIMIZATION FOR POINT-TO-POINT MOTION}

According to [17] it is possible to distinguish two approaches for the leg trajectory planning. The first is a Cartesian space-oriented approach, where the desired trajectory of a leg end point is set, and then the trajectories for each joint are identified by applying a transformation between the Cartesian and end point coordinates (2). This approach is a natural choice in applications where obstacle avoidance is the primary concern. However, control algorithms are developed for the joint coordinates due to the absence of a sensor capable of measuring the position of a leg end-point in the Cartesian space. Moreover physical constraints of the joint actuators such as torque, speed and voltage are defined for each motor, so the second, joint-space-oriented approach is more widespread in industry in general and for a robot leg movement in particular. The trajectory is planned in the following way; first, intermediate waypoints are chosen in the Cartesian space, then these points' coordinates are transferred to the joint space through (2), further interpolation between the intermediate waypoints in the joint space is performed by some polynomial function of time. While choosing the order of the polynomial, the main focus is on making the position and angular speed match the intermediate waypoints. Moreover, smooth operation is required, so polynomials of a higher degree $\left(5^{\text {th }}, 7^{\text {th }}\right)$ are normally used or their combination.

In this paper the focus is on minimization of the motor power consumption so the second approach for the reference trajectory generation is adopted. The trajectory is developed in order to minimize the actuators' DC-motors copper loss (it is assumed that the copper loss dominates for the motors in robotic applications) whilst ensuring that it passes through the intermediate waypoints identified in the Cartesian space. 


\section{A. DC-motor approximation as a dual-integrator system}

Each joint is equipped with a DC motor, so in order to find the trajectory which minimizes motor copper loss $\left(P_{\text {loss }}=\right.$ $i_{a}^{2} R_{a}$ ), a motor model first should be obtained [18].

$$
\begin{cases}\frac{d i_{a}}{d t}=-\frac{R a}{L_{a}} i_{a}-\frac{\lambda_{f}}{L_{a}} \omega_{r m}+\frac{1}{L_{a}} v_{a} & \left|i_{a}\right| \leq I_{\max } \\ \frac{d \omega_{r m}}{d t}=\frac{\lambda_{f}}{J_{e}} i_{a}-\frac{B_{f r}}{J e} \omega_{r m}-\frac{1}{J e} T_{L} & \left|v_{a}\right| \leq V_{\max } \\ \frac{d \theta_{r m}}{d t}=\omega_{r m} & \end{cases}
$$

where $i_{a}$ and $v_{a}$ are the armature current and voltage. $R_{a}, L_{a}$ and $\lambda_{f}$ are motor electrical parameters, representing the stator winding resistance, inductance and back-EMF constant respectively. $\theta_{r m}$ and $\omega_{r m}$ are rotor position and speed, $J_{e}$ and $B_{f r}$ are the total inertia and friction coefficient respectively. $T_{L}$ is the shaft load torque, which is considered to be equal to zero during the leg swing phase or could be identified through the procedure described in [13] during the support phase. $I_{\max }$ and $V_{\max }$ represent the inverter maximum current and voltage, representing the electrical limits of the leg actuator operation.

The scope of this paper is to identify the optimal transient behaviour of a drive, while in the joint space moving the robot foot from the initial to the end point without the study of the forces (torques) resulting from the ground reaction of the foot, so the load torque $T_{L}$ from equation (3) is set to zero throughout the discussion. Moreover due to the assumption that copper loss dominates over all other losses, the friction coefficient $B_{f r}$ is set to zero for simplicity. The electrical time constant of a drive is significantly smaller than the mechanical one, so according to [19] the DC motor can be approximated as a double integrator system presented in (4).

$$
\left\{\begin{array}{cl}
\dot{x}_{1}=x_{2} & |u| \leq \lambda_{f} I_{\max } / J_{e} \\
\dot{x}_{2}=u & \left|x_{2}\right| \leq V_{\max } / \lambda_{f}
\end{array}\right.
$$

where $x_{1}$ is the joint space position, $x_{2}$ is the joint angular speed and $u$ is the joint acceleration, referred to the motor. From (4) the maximum acceleration $(A)$ which is possible to get from the motor with no external load depends on the maximum allowed armature current $\left(I_{\max }\right)$.

\section{B. Optimal energy control}

Given the joint description in a double integrator form (4), the task is to find the acceleration profile which minimizes the copper loss. The optimization problem, similar to one discussed in [20], is formulated in Table II, where $t_{0}=0$ and

\begin{tabular}{|c|c|c|}
\hline State equations & Boundary conditions & Physical constraints \\
\hline $\begin{array}{l}\dot{x}_{1}=x_{2} \\
\dot{x}_{2}=u\end{array}$ & $\begin{array}{c}x\left(t_{0}\right)=\left[\begin{array}{c}-X \\
0\end{array}\right], x\left(t_{f}\right)=\left[\begin{array}{l}0 \\
0\end{array}\right] \\
\text { Final time } t_{f} \text { - fixed }\end{array}$ & $|u| \leq A$ \\
\hline \multicolumn{3}{|c|}{ Performance measure } \\
\hline \multicolumn{3}{|c|}{$\mathcal{J}_{e}=\frac{1}{2} \int_{t_{0}}^{t_{f}} u^{2}(t) d t \rightarrow \underbrace{\min }_{u(t)}$} \\
\hline
\end{tabular}

TABLE II. CONTROL CONSTRAINED MINIMUM ENERGY PROBLEM

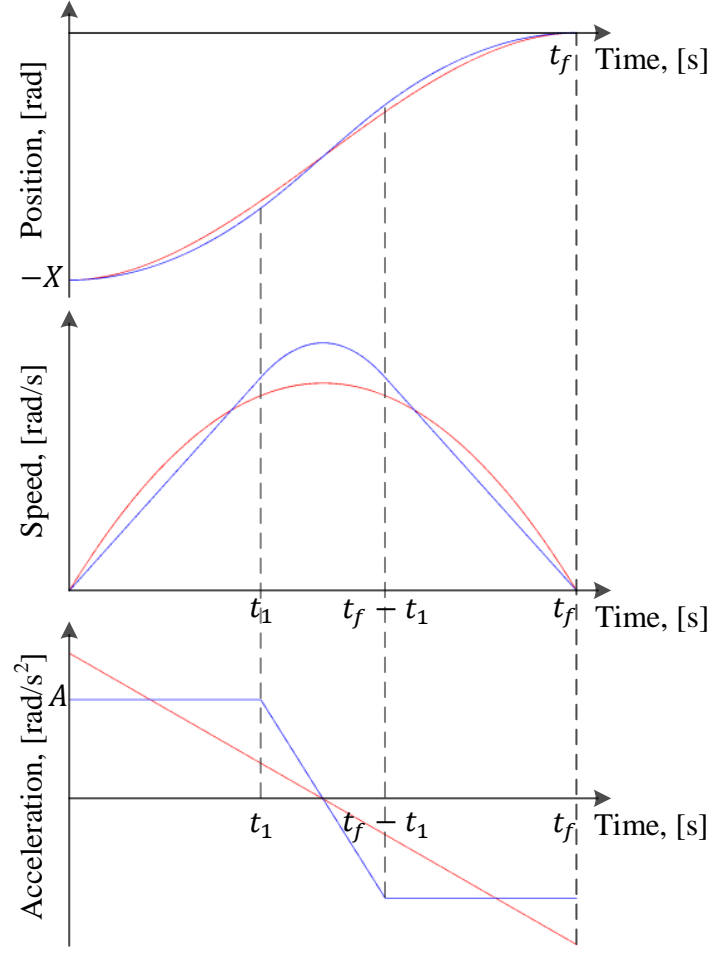

Fig. 3. Optimal energy state and control trajectories.

$t_{f}$ are initial and final time respectively and $X$ is the required movement distance, in other words $X$ represents the angular rotation of the joint, which is less than 1 cycle for the hexapod, but since geared actuators are being used for the CORIN design, the actual motor will make a number of full rotations. Note that in Table II the initial position value is taken with a negative sign; this is done purely for the convenience of control law development, where both final position and speed are set to zero and initial position is set as negative value corresponding to the original desired travel distance. Speed constraint is omitted because for particular CORIN joint actuators the speed limit is never reached.

The problem specified in Table II can be solved by applying Pontryagin's minimum principle, described in [21], where the optimal energy solution for each particular drive in the CORIN leg with the specified movement time, $t_{f}$, can be found as

$$
u^{\star}(t)=-\frac{12 X}{t_{f}^{3}} t+\frac{6 X}{t_{f}^{2}}
$$

if $t_{f} \geq \sqrt{\frac{6 X}{A}} \quad$ or when the control constraints (acceleration/current limit) are not reached. For the constrained control case, the optimal energy solution is given in (6).

$$
u^{\star}(t)= \begin{cases}-A, & t>t_{f}-t_{1} \\ \frac{-2 A}{\sqrt{3 t_{f}^{2}-12 \frac{X}{A}}} t+\frac{A t_{f}}{\sqrt{3 t_{f}^{2}-12 \frac{X}{A}}}, & t_{1} \leq t \leq t_{f}-t_{1} \\ A, & t<t_{1}\end{cases}
$$


where $t_{1}=\frac{t_{f}}{2}-\frac{1}{2} \sqrt{3 t_{f}^{2}-12 \frac{X}{A}}$. For the both cases, the optimal energy trajectories for a double integrator, representing the single joint behaviour are shown on Fig. 3. The red curves represent the unconstrained movement, and the blue ones, when the maximum motor current or acceleration is reached.

\section{ENERGY REQUIREMENTS}

In the previous sections it was mentioned that only copper loss is taken in account while identifying the optimal control strategy, however different origins of current passing through the motor armature winding and resulting in a copper loss were not identified. It is possible to roughly split the torque (i.e. current) produced by the joint motors into three groups:

1. Maintain the body position in the support phase $\left(Q_{s p}\right)$,

2. Hold the leg weight during the transfer phase $\left(Q_{t p}\right)$,

3. Actuator acceleration and deceleration at no-load condition, during both support and transfer phases $\left(Q_{n l}\right)$.

Only the last torque component was considered in the optimal energy control derivation, because the load torque, $T_{L}$, was set to zero, but for the complete optimal energy solution the influence of each component should be evaluated.

The energy consumed by each of CORIN's leg during the support phase (assuming a tripod gait, three legs are in support phase while the other three are in transfer phase) could be roughly approximated as follows.

$$
Q_{s p}=\int_{t_{0}}^{t_{f}}\left(\frac{\frac{M}{3} l_{2} g}{N_{2} \lambda p m_{2}}\right)^{2} R_{2} d t
$$

where the subscript ${ }_{2}$ is devoted to the second joint motor, $N$ is an actuator gear ratio, $R$ actuator armature resistance and $\frac{M}{3}$ is the third part of overall robot weight, assuming that it is equally shared between the legs in a support phase. In a similar way the energy consumed during the transfer phase of each leg will take the same form as (7), but substituting instead of body mass, the weight of a single leg excluding the weight of the first joint. The consumed energy related to the torque required for the joint angle control could be found by integrating over time the square of the acceleration profiles shown on Fig. 3 with the corresponding coefficients.

Taking into account the robot parameters from Table I,III, the contribution of all three aforementioned components to the overall energy consumption was roughly estimated as $95 \%, 4 \%$ and $1 \%$ respectively, so the energy consumed to maintain the body position is dominant, making it crucially important to minimise $Q_{s p}$ instead of the energy consumed for the leg position change. In other words the performance measure from Table II should be changed to the following one.

$$
\mathcal{J}_{e}=\frac{1}{2} \int_{t_{0}}^{t_{f}}\left(\mu+u^{2}(t)\right) d t
$$

where $\mu$ is a parameter representing $Q_{s p}$. The solution of an optimization problem defined in Table II with the performance measure defined as (8) is known in the literature [21] and a simple closed-form solution exists, but only for a free final time $t_{f}$. If final time could be set free and $\mu \gg u^{2}(t), \forall t$ then in the performance measure (8) the second term of sum could be omitted, leading to (9), which is the optimal time (OT) form of the performance measure.

$$
\mathcal{J}_{t}=\mu \int_{t_{0}}^{t_{f}} d t=\int_{t_{0}}^{t_{f}} d t \rightarrow \underbrace{\min }_{u(t)}
$$

\section{A. Optimal time control though sliding mode control}

The solution of the optimal control problem defined in Table II, with performance measure (9), is well established in control literature [21],[19]. Using Pontryagin's minimum principle the close loop solution can be obtained.

For every time instant the OT control action could be identified corresponding to the position of the current state in the $x_{1}-x_{2}$ plane, shown on Fig. 4. If the initial point $\boldsymbol{x}\left(t_{0}\right)$ lies somewhere on segment $D-0$ of the $x_{1}-x_{2}$ plane, than the $u^{\star}(t)=+A$ control is applied and the system reaches the origin in a minimum time. Similarly, if the initial point $\boldsymbol{x}\left(t_{0}\right)$ lies on segment $B-0$, then the $u^{\star}(t)=-A$ and the system reaches the origin. In all other points of the $x_{1} x_{2}$ plane, in order to reach the origin, first some control should be applied to reach one of the segments $D-0$ or $B-0$, after which the previously mentioned control strategy is applied. For any initial condition from the left hand side of $D-0-B$ curve the way to reach the curve is to apply $u^{\star}(t)=+A$, so the segment $B-0$ is reached, where the optimal control should change the sign to $u^{\star}(t)=-A$ and state slides to the origin. A similar strategy is applied for the initial states from the right hand side of $D-0-B$ curve and sliding to the origin is performed along the $D-0$ segment. The line $D-0-B$ is known as a switching curve $s(\boldsymbol{x}(t))$, where the optimal control changes its sign.

$$
s(\boldsymbol{x}(t)) \triangleq x_{1}(t)+\frac{1}{2 A} x_{2}(t)\left|x_{2}(t)\right|
$$

The close loop optimal time control law for a double integrator system is formulated in (11). By applying (11) the robot is moving at maximum speed, within the joints acceleration/current limit, so the dominant component of energy consumption, $Q_{s p}$, is reduced.

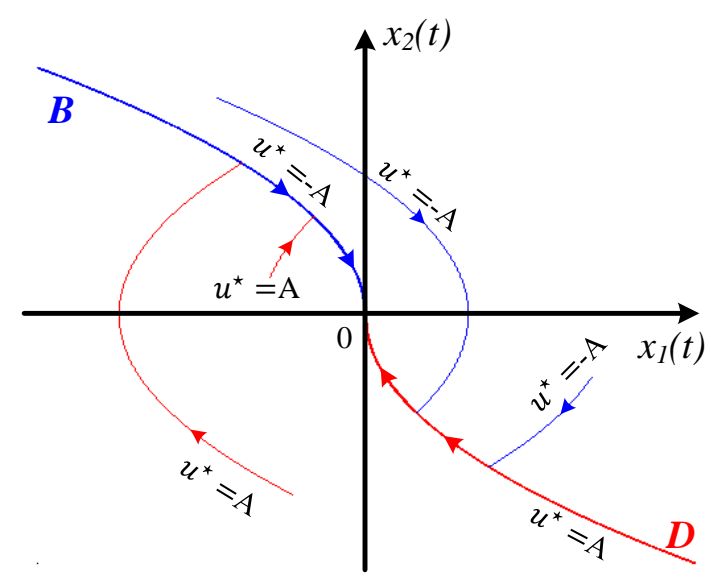

Fig. 4. Optimal time switching curve and state trajectories.[21] 


$$
u^{\star}(\boldsymbol{x}(t))=\left\{\begin{array}{l}
-A, \quad \forall \boldsymbol{x}(t): s(\boldsymbol{x}(t))>0 \\
+A, \quad \forall \boldsymbol{x}(t): s(\boldsymbol{x}(t))<0 \\
-A, \quad \forall \boldsymbol{x}(t): s(\boldsymbol{x}(t))=0, \quad x_{2}(t)>0 \\
+A, \quad \forall \boldsymbol{x}(t): s(\boldsymbol{x}(t))=0, \quad x_{2}(t)<0 \\
0, \text { for } \boldsymbol{x}(t)=0 .
\end{array}\right.
$$

\section{EXPERIMENTAL RESULTS AND DISCUSSION}

To evaluate the proposed robot joints control strategy it is compared with the Cartesian-space approach conventionally adopted for CORIN, where, at first, four intermediate waypoints are defined in Cartesian space as shown in Fig. 5, in order to prevent the feet being dragged across the floor. Then the Reflex model is used to generate a polynomial spline path, which is constrained by initial and final positions and initial and final speeds. Since four independent parameters exists, the cubic polynomial trajectory is chosen for a leg trajectory generation.

For the CORIN robot, MX series actuators by Robotis are used, with an integrated PID speed and position controller and Maxon motors (joints 1 and 3 are using MX-28T, while joint 2 uses MX-64T). The actuator parameters are presented in Table III.

In the control problem, defined in Table II, the control input or acceleration was limited to $A$, which represents either the current limit of an actuator or the maximum joint acceleration. In the experimental work the maximum current in the motor armature windings is not available, and the proportion of torque to support the body weight, the leg weight or to make a leg transfer is not identified, so the control action is limited simply by the maximum safe acceleration to prevent damage to the robot.

In order to compare the performance of different walking patterns from the energy point of view, a performance index called specific resistance was used [22], defined as the ratio of the total energy consumed for a travel to the travel distance, mass of a robot and gravitational acceleration. In this paper the following method is adopted: the travel distance is fixed, and the robot mass is kept the same, so it is valid to compare the energy consumed in the conventional and proposed method. For both walking patterns the stroke length and step height is kept the same. Joint acceleration and joint reference speed for the conventional and the proposed cases are shown in Fig. 6. It

TABLE III. CORIN ACTUATORS PARAMETERS

\begin{tabular}{|l|l|l|l|l|}
\hline Actuator & \multicolumn{4}{|l|}{} \\
\hline \multirow{4}{*}{ MX-28T } & Nominal voltage, $[\mathrm{V}]$ & 12 & $\begin{array}{l}\text { Torque constant, } \\
{[\mathrm{Nm} / \mathrm{A}]}\end{array}$ & $11.2 \mathrm{e}-3$ \\
\cline { 2 - 5 } & Nominal current, $[\mathrm{A}]$ & 0.413 & $\begin{array}{l}\text { Rotor inertia, } \\
{\left[\mathrm{kgm}^{2}\right]}\end{array}$ & $9.93 \mathrm{e}-8$ \\
\cline { 2 - 5 } & Terminal resistance, $R_{1,3}[\Omega]$ & 11.4 & Max. speed, $[\mathrm{rpm}]$ & 11000 \\
\cline { 2 - 5 } & Terminal inductance, $[\mathrm{mH}]$ & 0.343 & Gear ratio, $N_{1}$ & $193: 1$ \\
\hline \multirow{4}{*}{ MX-64T } & Nominal voltage, $[\mathrm{V}]$ & 9 & $\begin{array}{l}\text { Torque constant, } \\
{[\text { Nm/A] }}\end{array}$ & $8.55 \mathrm{e}-3$ \\
\cline { 2 - 5 } & Nominal current, $[\mathrm{A}]$ & 0.72 & $\begin{array}{l}\text { Rotor inertia, } \\
{\left[\mathrm{kgm}{ }^{2}\right]}\end{array}$ & $2.21 \mathrm{e}-8$ \\
\cline { 2 - 5 } & Terminal resistance, $R_{2}[\Omega]$ & 2.21 & Max. speed, $[\mathrm{rpm}]$ & 12000 \\
\cline { 2 - 5 } & Terminal inductance, $[\mathrm{mH}]$ & 0.085 & Gear ratio, $N_{2}$ & $200: 1$ \\
\hline
\end{tabular}

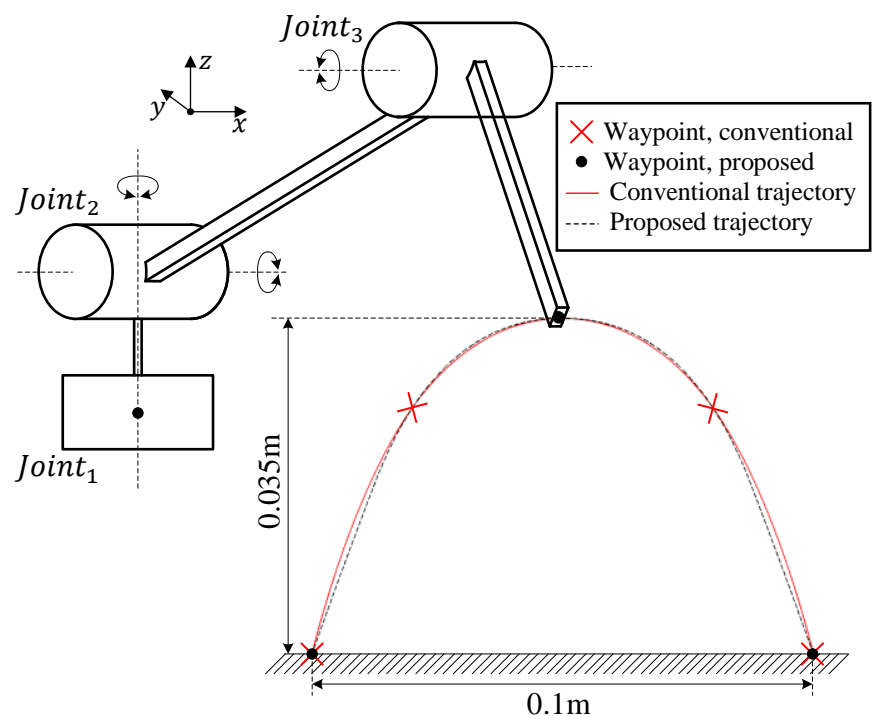

Fig. 5. Conventional and proposed leg trajectory.
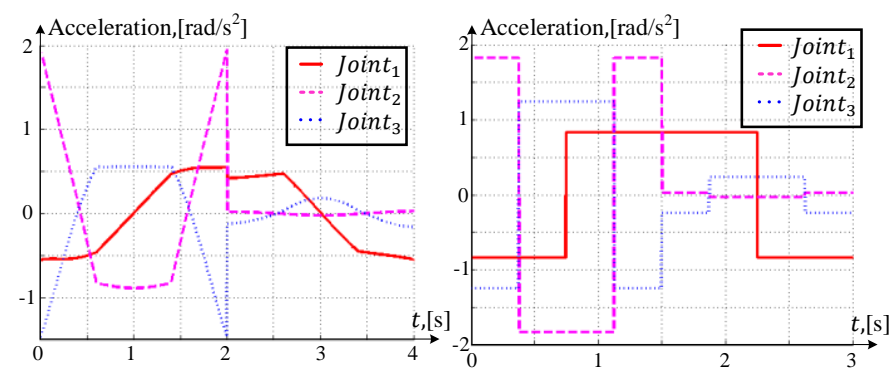

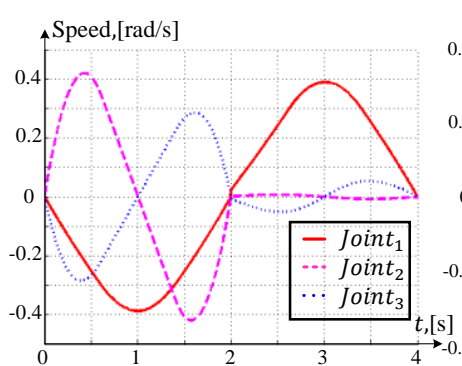

(a)

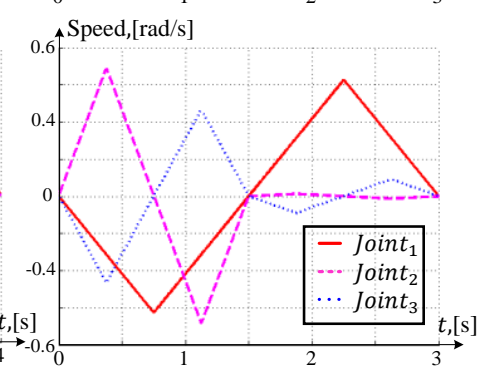

(b)
Fig. 6. Joint space acceleration and speed profiles: a) Conventional strategy b) Proposed strategy.

could be seen that the single step time is reduced by $25 \%$.

To verify the superiority of the proposed robot control, it was connected to the external DC power supply through a Yokogawa WT3000 power analyser, which was used to measure the electrical energy consumed during the whole locomotion. The measurement results for a three metre robot motion are presented in Table IV. In the proposed method the speed of locomotion is increased, so to move for $3 \mathrm{~m}, 3$ minutes instead of 4 is required compared to the conventional control method.

Three sets of experiments were conducted. The first measured the standby energy, $Q_{s b}$, consumed in the motor control PCBs; as expected, it reduces proportionally to the operating time reduction. In the second, the robot was placed on a support bar, preventing the robot legs from touching the 
TABLE IV. CORIN ENERGY CONSUMPTION

\begin{tabular}{|c|c|c|c|c|c|}
\hline & \multicolumn{2}{|c|}{ Original } & \multicolumn{2}{|c|}{ Proposed } & \multirow{2}{*}{$\begin{array}{c}\text { Saved } \\
\text { energy, } \\
\%\end{array}$} \\
\hline & Energy, mWh & $\%$ & Energy, mWh & $\%$ & \\
\hline $\begin{array}{l}\text { Stand by power } \\
\text { consumption }\end{array}$ & 446.6 & 30.6 & 336.9 & 30.2 & 24.6 \\
\hline $\begin{array}{l}\text { No support } \\
\text { phase }\end{array}$ & 660.8 & 45.3 & 504.8 & 45.3 & 23.8 \\
\hline $\begin{array}{l}\text { Full power } \\
\text { consumption }\end{array}$ & 1460 & 100 & 1115 & 100 & 23.6 \\
\hline
\end{tabular}

ground. In this experiment the $Q_{s p}$ is zero and measured energy consists of $Q_{t p}, Q_{n l}$ and $Q_{s b}$. The proposed algorithm shows almost $24 \%$ energy reduction, where only one third of this saving is due to the reduction of energy needed to hold the legs in the air, showing that the stand-by power consumption is very considerable in the CORIN design, making the proposed control approach even more significant. The last measurements were done for the real hexapod movement for $3 \mathrm{~m}$ on a flat and undistorted terrain. The overall energy consumption was reduced by more than $20 \%$ proving the supremacy of the proposed control strategy. Energy savings are achieved mainly due to the reduction of robot operating time, for the same distance travelled, with reduction during the transfer and support phases and for standby operation.

\section{CONCLUSION}

In this paper energy minimization strategy has been described for a mobile legged robot. The strategy minimizes the consumed energy of each joint in all robot legs by optimizing the joint reference trajectory. It was shown that the energy needed to maintain the robot body position, together with the robot electronics energy are the biggest parts of the overall energy consumption. Since both are dependent only on operation time the joint reference trajectory maximizing the robot speed was chosen. Experimental results show the 23.6\% energy efficiency improvement, making the proposed control very important for the robots operating off battery supplies, which is always true for mobile robots.

\section{REFERENCES}

[1] H. Khan, J. Iqbal, K. Baizid, and T. Zielinska, "Longitudinal and lateral slip control of autonomous wheeled mobile robot for trajectory tracking," Frontiers of Information Technology \& Electronic Engineering, vol. 16, pp. 166-172, 2015

[2] C. H. Yoo, "Self-driven robotic cleaning apparatus and driving method thereof," ed: Google Patents, 1994.

[3] "NASA's Mars Exploration Program Website," https://mars.nasa.gov/.

[4] M. H. Raibert, Legged robots that balance: MIT press, 1986.
[5] S.-M. Song and K. J. Waldron, Machines that walk: the adaptive suspension vehicle: MIT press, 1989.

[6] K. Nagatani, S. Kiribayashi, Y. Okada, K. Otake, K. Yoshida, S. Tadokoro, et al., "Emergency response to the nuclear accident at the Fukushima Daiichi Nuclear Power Plants using mobile rescue robots," Journal of Field Robotics, vol. 30, pp. 44-63, 2013.

[7] S.-K. Chu and G.-H. Pang, "Comparison between different model of hexapod robot in fault-tolerant gait," IEEE Transactions on Systems, Man, and Cybernetics-Part A: Systems and Humans, vol. 32, pp. 752 756, 2002.

[8] N. Paine, S. Oh, and L. Sentis, "Design and control considerations for high-performance series elastic actuators," IEEE/ASME Transactions on Mechatronics, vol. 19, pp. 1080-1091, 2014.

[9] A. G. Gonzalez-Rodriguez, A. Gonzalez-Rodriguez, and F. CastilloGarcia, "Improving the energy efficiency and speed of walking robots," Mechatronics, vol. 24, pp. 476-488, 2014.

[10] D. C. Kar, "Design of statically stable walking robot: a review," Journal of Field Robotics, vol. 20, pp. 671-686, 2003.

[11] D. W. Marhefka and D. E. Orin, "Gait planning for energy efficiency in walking machines," in Robotics and Automation, 1997. Proceedings. 1997 IEEE International Conference on, 1997, pp. 474-480.

[12] S. S. Roy and D. K. Pratihar, "Dynamic modeling, stability and energy consumption analysis of a realistic six-legged walking robot," Robotics and Computer-Integrated Manufacturing, vol. 29, pp. 400-416, 2013.

[13] S. S. Roy and D. K. Pratihar, "Effects of turning gait parameters on energy consumption and stability of a six-legged walking robot," Robotics and Autonomous Systems, vol. 60, pp. 72-82, 2012.

[14] N. Kottege, C. Parkinson, P. Moghadam, A. Elfes, and S. P. Singh, "Energetics-informed hexapod gait transitions across terrains," in Robotics and Automation (ICRA), 2015 IEEE International Conference on, 2015, pp. 5140-5147.

[15] D. W. Marhefka and D. E. Orin, "Quadratic optimization of force distribution in walking machines," in Robotics and Automation, 1998. Proceedings. 1998 IEEE International Conference on, 1998, pp. $477-$ 483.

[16] S. S. Roy, A. K. Singh, and D. K. Pratihar, "Estimation of optimal feet forces and joint torques for on-line control of six-legged robot," Robotics and Computer-Integrated Manufacturing, vol. 27, pp. 910-917, 2011.

[17] K. Fu, R. Gonzalez, and C. Lee, "Robotics: Control," Sensing, Vision and Intelligence, pp. 149-200, 1987.

[18] S.-K. Sul, Control of electric machine drive systems vol. 88: John Wiley \& Sons, 2011

[19] F.L. Chernousko, I.N. Ananevskiy, and S.A. Reshmin, Memodbl управления нелинейными механическими системами (Nonlinear mechanical systems control methods): Litres, 2016.

[20] A. Bodrov, E. Poupard, W. P. Heath, and J. Apsley, "Minimum electrical energy use in a multi-actuator system," in Electric Machines and Drives Conference (IEMDC), 2017 IEEE International, 2017, pp. 16

[21] D. E. Kirk, Optimal control theory: an introduction: Courier Corporation, 2012.

[22] B. Siciliano and O. Khatib, Springer handbook of robotics: Springer, 2016. 